\title{
INTERMITENCIAS DE LA VERDAD: UNA APROXIMACIÓN A LAS TECNOLOGÍAS DE INFORMACIÓN Y COMUNICACIÓN DESDE DIVERSOS PARADIGMAS DE LA CIENCIA
}

\author{
INTERMITENCIES OF THE TRUTH: AN APPROXIMATION \\ TO INFORMATION \\ AND COMMUNICATION TECHNOLOGIES FROM \\ DIFFERENT PARADIGMS OF THE SCIENCE
}

Marymili Segura Vera ${ }^{24}$

\section{RESUMEN}

El presente escrito tiene como finalidad analizar la evolución de las Tecnologías de Información y Comunicación (TIC) como parte de la verdad aceptada por la ciencia en diferentes momentos de la historia occidental, así como los fundamentos y criterios de validez que han enmarcado el desarrollo de las tecnologías desde la perspectiva de diversos paradigmas científicos. Para el desarrollo de este trabajo se realizó un análisis documental. Se concluye que la verdad, al igual que las TIC, cambia de acuerdo a los intereses, al enfoque que se quiera dar. La intermitencia de la verdad es lo que la hace apasionante y hace necesarios los criterios de validez científica, con los que nos permitimos consolidar ese destello -esa verdad- que a uno a varios interesa resaltar.

Palabras Clave: Epistemología y TIC; Conocimiento y Tecnología; Paradigmas y TIC

\begin{abstract}
The purpose of this paper is to analyze the evolution of Information and Communication Technologies (ICT) as part of the truth accepted by science at different times in Western history, as well as the fundamentals and validity criteria that have framed the development of technologies from the perspective of various scientific paradigms. For the development of this work a documentary analysis was carried out. It is concluded that the truth, like ICT, changes according to interests, to the approach you want to give. The intermittency of the truth is what makes it thrilling and makes necessary the criteria of scientific validity, with which we allow us to consolidate that flash - that truth - that one to several interests to highlight.
\end{abstract}

Keywords: Epistemology and ICT; Knowledge and Technology; Paradigms and ICT

24 Lcda. en Educación, Magister en Educación Mención Investigación Educativa, Especialista en Tecnologías de la Computación en Educación. Responsable del Módulo Virtual en Investigación, Facultad de Ciencias de la Educación de la Universidad de la Empresa. E-mail: marymilisegura@gmail.com 


\section{INTRODUCCIÓN}

La Verdad ha ocupado a muchos a lo largo de la historia, quizás por el simple hecho de que lo que ha marcado cada etapa en el tiempo es el cambio en la manera en que ésta se percibe; en la que se describe. Desde aquella luz fuera de la caverna a la que, según Platón, solo los virtuosos podían acceder; ubicándose siglos después en el Dios de los hebreos, la verdad absoluta existió por muchos años como un todo externo; como una meta en muchos pensadores. Aun no viéndose ni palpándose, era la verdad, porque fue el origen de todo lo que existe. Esa verdad plasmada por Dios en su creación. La creación era, pues, la verdad palpable. Verdad que se cayó para los científicos, pero sigue siendo la verdad, porque ellos han investigado y justificado aspectos de la creación, en función de la existencia de ese Dios.

Y podríamos preguntarnos ¿pero cómo? A pesar de que ya se ha demostrado que la creación tiene orígenes que no son divinos, por ejemplo, a través la teoría de la evolución... ¿Cómo aún se mantiene vigente la creencia en Dios?... Cuando una catástrofe natural inesperadamente sucede, aunque los científicos expliquen las razones por las que se originó, fue causada por una fuerza que hace que ese evento acontezca. Esa cosa sobrenatural es esa verdad que ninguno ve pero que sabemos que existe, que podríamos llamar la verdad escondida.

Por otra parte, ésta es otra verdad visible, medible, cuantificable que el hombre puede modificar y mejorar para su bienestar: la tecnología. Ésta que también ha existido desde la aparición del hombre, por la necesidad de hacer más sencillos y llevaderos los procesos básicos para su subsistencia como individuo y como ser social, y se ha mantenido cambiante, pero siempre presente a lo largo de la historia y explicada desde la perspectiva de las diferentes corrientes epistémicas.

A continuación, se esbozará brevemente la percepción de la verdad a lo largo de la historia del conocimiento, pasando por diferentes paradigmas científicos, sus criterios de validez y el abordaje de las tecnologías a través de éstos.

\section{LA VERDAD HASTA NUESTROS DÍAS}

La Verdad en la antigüedad y la edad media estaba sustentada en la tradición y la fe. Se plantea como eterna, permanente, original, absoluta, indiscutible, tal como lo expresa Santo Tomás de Aquino: "Es evidente que existe la verdad. Porque el que niega que existe la verdad, conoce que la verdad existe. Si, pues, no existe la verdad, es verdad que la verdad no existe". En razón de este dicho, se puede agregar que la verdad, como un todo, existe desde el momento de la creación y antes de la creación misma, ya que su fundamento principal de existencia es la fuerza de un Dios verdadero; de él nacen y nacerán todas las verdades conocidas y por conocer.

Por otra parte, Aristóteles desde su perspectiva y a través de la observación, da importancia a la explicación científica de las causas de esta verdad; indica así que hay cuatro tipos de causa: las dos primeras son intrínsecas la formal (estructura, su esencia); la material (contenido). Y las dos últimas son extrínsecas: final (función, intención; lo que produce un cambio o movimiento); la eficiente (ex - previo; la meta de un ser). Lo que da sentido a la necesidad de buscar explicaciones más profundas sobre la verdad. En este sentido Mardones (1994) expresa que "una explicación científica adecuada debe especificar estos cuatro aspectos que constituyen su causa. Sobre todo, no podía faltar a una pretendida explicación científica de un fenómeno o hecho, el dar cuenta de su canal final o telos" (p.22).

Entonces el científico, observador, hurga o válida hasta dónde quiere llegar. La verdad llega hasta dónde quiere llegar el científico, el filósofo. Y cuando éste llega a esa verdad, está probando que existe. (la verdad moderna: provisional, relativa y crítica).

Así, a lo largo del tiempo, la percepción de la verdad pasó de ser algo externo, a ser algo que está en el sujeto. Desde el planteamiento de Descartes "primero pienso y luego existo"; o, desde la perspectiva de Hume "Si no partiésemos de un hecho presente en la memoria y en los sentidos, nuestros razonamientos serían meramente hipotéticos", la verdad es algo que se percibe del mundo que nos rodea y que deber ser comprobada a través de nuestros sentidos. Entonces, ¿la verdad como un todo está en el pensamiento o está en los sentidos?

Kant viene, pues, a plantear la verdad como aquello que podemos apreciar a través de la conjunción de nuestros sentidos y nuestros conocimientos, pero aclara que más allá de ellos está la verdad en sí misma, 
una a la que no accederemos (el noúmeno). Por lo que se busca la verdad de lo que a cada quien le interesa conocer y comprobar. En este sentido Kant dice que el conocimiento que tenemos del mundo exterior "no es un reflejo de las cosas, sino un producto de nuestro pensamiento excitado por la apariencia de las cosas, es decir por lo que se llama fenómeno". (Kant citado en Bergua 1970, p.93)

Para llegar a la verdad moderna hay que partir de un porqué y ese porqué es la verdad absoluta, esa que nadie conoce pero que imaginamos. Entonces a medida que el hombre se ha ido puliendo científica, filosófica y culturalmente, ha querido descubrir dentro de esa verdad absoluta la verdad que a él le interesa, y ha ido estudiando, analizando y buscando; usando tecnología para llegar a ella. Pudiendo inclusive ser la tecnología el fenómeno mismo, traducido en un objeto que es diseñado y luego creado por el hombre; pero también en la necesidad que surge del ser humano, de su pensamiento. La necesidad de que exista y se pueda utilizar ese objeto tecnológico puede tomarse como fenómeno, cómo la verdad que el hombre cree que está inventando, pero que en realidad está descubriendo porque ya existe.

De esta manera, dependiendo de los momentos históricos, los problemas abordados y de las matrices epistémicas, la dinámica del pensamiento siguió estableciendo concepciones diversas sobre la verdad, como es el caso de Hegel que a través de su llamado "movimiento dialéctico" la considera más bien el devenir entre ser y no ser. Desde su perspectiva de que "La contradicción es la raíz de todo movimiento y de toda vida y es solo en la medida en que contiene una contradicción que algo se mueve y tiene impulso y actividad" (Hegel en Dunayevskaya, 2004. p.36) realza la dialéctica en el proceso de la búsqueda de la verdad y con esto abre la puerta a otra manera de verla.

Al respecto, Selsam (1962) comenta sobre las tres expresiones que utilizó Hegel para representar el carácter dinámico del universo: La primera viene a ser "la unidad de los contrarios" el ser y la nada son contrarios y sin embargo cuando se unen constituyen algo, el devenir. La segunda la identifica como "negación de la negación", lo que trataba de demostrar era que una proposición enunciada de forma unilateral es negada por su propia contradicción interior, conduciendo a la afirmación de lo contrario, o sea a su negación. La tercera expresión utilizada por Hegel es que la cantidad se transforma en calidad, surgirá una contradicción entre la vieja forma y el nuevo contenido.

Esta manera de explicar el universo, hace percibir la verdad de un modo diferente y por demás fascinante; tal vez la necesidad de probar que la verdad del futuro no se parece a la verdad del presente, hace que surja una forma diferente de demostrarlo que estamos viviendo actualmente y de compararlo con lo que no es verdad todavía pero que ya se ha pensado, que otros se han imaginado y se han sentado a probar de forma científica. Considero que es ésta la base del surgimiento de todos los adelantos tecnológicos, pues esa contradicción entre la espiritualidad del ser y la sistemática inercia de la tecnología es lo que ha llevado a la evolución y perfeccionamiento de ambos a lo largo de la historia.

No obstante, tras ese motor que es la búsqueda de la verdad, que ha impulsado y a la vez limitado tantos descubrimientos en el devenir del tiempo, Nietzsche (en Prieto, 2002) define El <<mundo verdadero>> como "una idea que ya no sirve para nada, que ya ni siquiera es obligante, una idea que ha llegado a ser inútil y superflua, en consecuencia, una idea refutada" (p.66). Entonces, ¿Siendo una idea refutada, en ningún momento pudo ser verdad?, ¿Lo que se niega mediante análisis bien fundamentados deja de ser verdad y pasa a ser inexistente?, ¿Cómo saber que un planteamiento es verdaderamente válido? Quizás la necesidad de responder preguntas como éstas marcó el surgimiento de los diferentes paradigmas a través de los cuales se busca dar validez a lo que muchos llaman verdad científica.

\section{CRITERIOS DE VALIDEZ}

Siguiendo en esa necesidad de comprobar, ya no la verdad como un todo, sino la veracidad de un planteamiento, se busca justificarlo y fundamentarlo, entendiendo que a su vez esto conlleva el riesgo de que al ser validados puedan refutar los planteamientos de otros, pero potencialmente en un futuro podría éste nuevo planteamiento ser desvirtuado también. El establecimiento de la validez científica históricamente se ha logrado a través de métodos que, enmarcados en fundamentos filosóficos, epistemológicos y ontológicos permiten comprobar la veracidad o falsedad de dicho planteamiento.

Así, en la modernidad se instituye como base para establecer la validez científica el método hipotético deductivo -conocido también como método científico- asumiendo entonces que las "concepciones de conocimiento y ciencia imperantes se fundamentan en la observación y comprobación de lo real, es decir, de lo que 
existe dentro de las coordenadas de tiempo, espacio y masa" (Hurtado y Toro, 2005). Siendo esta tendencia hacia lo hipotético deductivo reforzada más adelante por Popper quien indica, según Mardones (1991), que el método científico es único en todo campo de investigación científica, es decir, todas las ciencias, teóricas o generalizadoras usan el mismo método, ya sea en ciencias naturales o en ciencias sociales. Y éste se desarrolla en forma triada: problema-conjetura-refutación (crítica). (p.90)

Sin embargo, entendiendo que la ciencia no solo necesita de condiciones económicas sino también de condiciones sociales para poder mantenerse, surge la concepción de paradigma como factor significante al momento de establecer la validez científica. Kuhn (en Pérez, 2006) expresa que "en la elección de un paradigma: no hay ninguna norma más elevada que la aceptación de la comunidad pertinente" (p.206) y para esto es importante la argumentación, necesaria para lograr la persuasión y aceptación dentro de estas comunidades científicas. Al respecto Mardones (1991) señala que "Un paradigma es lo que los miembros de una comunidad científica comparten, y, recíprocamente, una comunidad científica consiste en hombres que comparten un paradigma" (p. 199).

Con los planteamientos de Kuhn se abren puertas para ver la realidad y los avances científicos de manera diferente, dando entrada, entre otras cosas, a un análisis diverso de las consecuencias y los aspectos sociales que causan o que derivan de la misma ciencia. El fenómeno de la tecnología, sus avances, su creciente arraigo en todos los ámbitos de la sociedad y su incidencia en muchos cambios sociales pueden ser estudiados y validados científicamente gracias a la existencia de los diversos paradigmas científicos.

Así, han surgido diferentes paradigmas científicos que fueron consolidándose y sirviendo a través de sus métodos como base para establecer los distintos criterios de validez científica que conocemos en la actualidad:

En el caso del positivismo, a pesar de que el concepto positivista puede remontarse a los tiempos de David Hume y a la filosofía crítica de Emmanuel Kant, entre otros, fue Comté quien utilizó este término por primera vez en el siglo XIX (Moreno, 2003). Estableciendo el positivismo como una doctrina y, como señala Moya (1998), "siendo precisamente Comte responsable de los tres principios programáticos del positivismo contemporáneo, a saber: El principio empirista de significidad, el principio de la validación empírica de las teorías científicas y el principio de la conservación del desarrollo científico" (p.41)

El positivismo ve entonces el fenómeno desde afuera; desde este paradigma la verificación y el criterio del lenguaje demarcan lo que es ciencia y lo que no es; se busca entonces a través del lenguaje científico evitar la ambigüedad, enfocándose en los hechos y describiéndolos desde una objetividad ontológica y axiológica. Caracterizándose por el cientificismo y por enaltecer valores como el orden y el progreso.

A lo largo de la historia de los avances tecnológicos el positivismo ha dado grandes aportes, inclusive cuando se comienza a entender los efectos sociales que derivan del uso de la tecnología en sus diferentes acepciones. Siendo tantos los campos en los que está presente la tecnología y tan remota su historia, voy a limitarme de aquí en adelante a hablar de las tecnologías de información y comunicación (TIC) y de su evolución en las últimas 4 décadas. En este sentido desde el paradigma positivista se logró vislumbrar a nivel social muchas consecuencias intrínsecas en el uso de las TIC, llegaron a reforzarse hipótesis sobre las actitudes en el uso de las TIC en diversos grupos sociales, por ejemplo. Lo que llevó a la producción y uso masivo de muchos de los beneficios tecnológicos presentes en la actualidad.

El paradigma positivista, ciertamente, ha dado grandes frutos a la ciencia, sin embargo, la aparición del racionalismo crítico pone en tela de juicio ciertos aspectos del positivismo, estableciendo otros criterios para validar lo científico, como es el caso de la falsación. Así lo expone Popper (en Mardones, 1991), cuando resalta que:

Todos los experimentos pueden interpretarse como intentos de extirpar teorías falsas, de encontrar puntos débiles en una teoría para rechazarla si queda refutada por el experimento;(...) solo si no se pueden refutar a pesar de los esfuerzos se puede decir que ha superado bien severos experimentos. (p.92)

Al respecto Moya (1998), compara dogmatismo frente a actitud crítica como lo que para Popper mejor representaría las diferencias entre el modelo positivista y el racionalismo crítico. No obstante, a pesar de las diferencias que se plantean, el racionalismo crítico mantiene del positivismo el hecho de utilizar el método hipotético deductivo y el uso de un mismo método tanto para las ciencias sociales como para las ciencias naturales (Mardones, 1991) 
No se puede negar que la falsación ha impulsado muchos avances en la ciencia y la tecnología, precisamente en el mundo de las TIC se percibe con elevada frecuencia esta dinámica. Vemos como en pocos años la manera de comunicarnos ha cambiado y sigue cambiando vertiginosamente. Muestra de esto vienen a ser los teléfonos celulares, cada vez surge una versión nueva pretendiendo "ser la mejor"y en poco tiempo resultan ser falsas esas pretensiones, pues surgen celulares con nuevas tecnologías y características que refutan o dejan atrás las ya existentes.

Sin embargo, no todo puede ser explicado desde el positivismo o neopositivismo; o estudiado y validado a través de la observación y el método científico, hay situaciones que no pueden ser asumidas de forma literal o que deben ser contextualizadas teniendo en cuenta, no solo tiempo y espacio, sino también el sentir de los protagonistas y el significado del lenguaje utilizado.

Entonces, ¿cómo validar costumbres, creencias o sentimientos? Una manera es utilizando una técnica antigua pero que se mantiene y que se ha ido perfeccionando y formalizando hasta llegar a ser un enfoque a través del cual se pueden desarrollar estudios científicos: La Interpretación o Hermenéutica.

La Hermenéutica, que tuvo su origen en el análisis textual, principalmente de los libros bíblicos, fue adoptada por Dilthey como base metodológica en un intento por dar a las ciencias humanas la precisión y certeza que habían caracterizado el éxito y prestigio de las ciencias naturales. Así los textos y los productos culturales llegaron a ser para Dilthey la llave para la comprensión de las intenciones subjetivas y la vida histórica de sociedades remotas (Ulin,1990)

Por otra parte, Gadamer enfoca el proceso hermenéutico en una relación circular. "El acuerdo de todas las partes con el todo es el criterio permanente para la rectitud de la comprensión" (Mardones, 1991. P.108). En el aspecto social, para entender un hecho específico se debe interpretar, no solo el lenguaje que utilizan los involucrados, sino sus costumbres, su contexto y comprender la incidencia de cada uno de esos aspectos en el hecho o fenómeno a estudiar. En sí con la hermenéutica se logra llegar más allá en el estudio de las ciencias humanas, pues se hace posible interpretar lo individual con respecto a su contexto.

Es bien sabido que las TIC en la actualidad forman parte de un proceso social; al respecto, Adell (2007) plantea que éstas tecnologías están cambiando nuestro sistema educativo, cómo trabajamos, cómo nos relacionamos unos con otros, cómo pasamos nuestro tiempo libre y, en suma, nuestros modos de percibir y relacionarnos con la realidad y a nosotros mismos. En consecuencia, como todo proceso social muchos aspectos relacionados con el uso de las TIC no pueden tomarse de forma objetiva o literal, sino que deben interpretarse según el contexto y la perspectiva de la o las personas involucradas, para lo cual es importante tener presente los fundamentos de la hermenéutica.

Con respecto al paradigma interpretativo es importante también tener en cuenta el gran aporte dado por Husserl a través de la fenomenología, método que para Serrano (2007) "permite la elaboración de una filosofía como ciencia eidética, es decir, como ciencia de las esencias" (p. 22) y que según Díaz (2011), "consiste en sustraer la intencionalidad de la conciencia de la esfera de la experiencia psíquica y llevarla a la esfera de la pura validez lógico-objetiva" (p.93). Las ideas y enseñanzas de Husserl influenciaron a muchos estudiosos entre quienes se encuentran M. Heidegger, H. G. Gadamer, E. Levinas, J. P. Sartre, H. Marcuse, K. Lowit, L. Landgrebe, entre otros.

Sin embargo, una de las características del paradigma interpretativo es que su criterio de validez no está basado en un solo método, sino que se apoya en varios, como los mencionados por Díaz (2011): "El etnográfico, El fenomenológico, Fenomenológico Hermenéutico, Etnometodología, Interaccionismo Simbólico, Estudio de Casos (Historia de Vida), Teoría Fundamentada, Documental (Análisis de contenido)" (p.39).

Pero ¿y al momento de estudiar el contexto en sí?, ¿Cómo analizar la dinámica de los movimientos sociales desde su esencia, no individual sino de grupo? Siguiendo en la línea de las TIC, ¿cómo estudiar y obtener resultados formales de la incidencia de éstas en los cambios de actitudes y conductas de los diferentes grupos sociales?

Una alternativa para abordar estas necesidades es la Teoría Crítica que, según Muñoz (2000), tiene su origen en la escuela de Frankfurt consecuencia de los acontecimientos en Europa en la segunda década del siglo XX, buscando desarrollar una reflexión sobre los procesos que consolidaban la sociedad burguesacapitalista y todo el significado social que esto conllevaba. En los proyectos de la Escuela de Frankfurt estuvieron involucrados, entre otros, Horkheimer, Adorno, Benjamin, Fromm y Marcuse. Y es después de unos años que Horkheimer le da a este movimiento la denominación de Teoría crítica. 
Esta corriente epistémica, teniendo sus inicios en la Dialéctica de la ilustración, enfoca su metodología en el estudio de los procesos sociales y centra su razón de ser en la lucha contra la dominación, criticando la concepción presentada desde la Racionalidad Instrumental de que todo orden social convierte a los seres humanos en instrumento. Al respecto, Marcuse (en Moya, 1998) expresa que "la racionalidad tecnológica protege así, antes que niega, la legitimidad de la dominación y el horizonte instrumentalista de la razón se abre a una sociedad racionalmente totalitaria" (p.186). Criticando de esta manera el hecho de que la meta se traduzca simplemente en lograr producir más en menos tiempo, sin importar las necesidades de las personas involucradas sino lo práctico, lo que genera ganancias y aumenta capitales.

Ante todo este panorama, Habermas presenta una visión menos pesimista; habla de la "Racionalidad Comunicativa" en donde, tomando en cuenta la sociedad, la representa como un conjunto de sistemas complejos y estructurados de proceso donde el individuo puede pasar de ser solo un elemento más de estos procesos, a ser el creador principal de los mismos sumergido en la subjetividad de los significados del mundo de vida y enmarcado todo esto en la comunicación.

Al respecto, Habermas, según Fernández (1997), propone su propia versión de la crítica a la sociedad y renovadas alternativas de superación de las controversias mediante su concepto de acción comunicativa, entendiéndose esta como "fundamentación metodológica de las Ciencias Sociales en una Teoría del Lenguaje"; y su ética del discurso en la que, según el mismo autor, para Habermas la situación formal de la argumentación discursiva con otros sigue ciertas reglas las cuales son detalladas "en lo que él llama la situación ideal del habla y esto lleva al resultado de una ética de procedimiento"

Así pues, la respuesta del grupo social a través de la reflexión, el cambio, la transformación, la valoración, la toma de decisiones y la emancipación es la que darán validez a las teorías, siendo uno de los métodos utilizados la investigación acción participativa (Díaz, 2011). Desde esta perspectiva epistemológica, y al igual que en otros campos de acción, se pueden lograr muchos avances en el uso e implementación de aplicaciones tecnológicas partiendo de las necesidades y tradiciones que surjan de los grupos de usuarios involucrados.

Sin embargo, en esa dinámica constante de la investigación y la ciencia surge otro paradigma que permite comprender situaciones o problemas desde una óptica diferente, se trata de la complejidad que, según Moreno (en Velilla, 2002):

Desde sus principios holográmico y de recursión ofrece un marco conceptual que presupone una visión no lineal, no mecánica y no acumulativa de cualquier desarrollo. Así, esta teoría coincide en sus presupuestos con la epistemología contemporánea, pero la mayor aportación de las teorías de complejidad podría estar en el lugar en donde se agota la epistemología contemporánea, es decir, en lo concreto de esa no linealidad, o en lo especial y curiosa de la lógica de ese desarrollo. (p.122)

Entonces desde la complejidad se permite abordar los problemas minimizando los parcelamientos paradigmáticos, surgiendo con esto nuevos criterios de validez científica. No obstante, la percepción de la verdad sigue su intermitente camino de atinos y desatino en el que surgen nuevos contextos y nuevos aspectos a tener en cuenta para llegar a ella a través de la investigación y la ciencia, aspectos que en otros tiempos fueron quizás invisibles van surgiendo en la actualidad, se perciben cada vez con más intensidad y destellando ante los ojos de la ciencia su relevancia; aspectos como el espíritu, las creencias, la cultura pasan a ser determinantes al momento de comprender la realidad actual y las necesidades de la humanidad en todos los campos, siendo uno de los principales el tecnológico.

En el marco de esta dinámica búsqueda de la verdad emerge el enfoque Integral Holónico el cual, según Wilber (2006), radica en una visión integrada de la realidad en la que el acceso al conocimiento dispone de tres vías que el identifica como: el ojo de la carne, a través del que se percibe el mundo externo, el tiempo y los objetos; el ojo de la mente, que permite alcanzar el conocimiento de la filosofía de la lógica y de la mente; y el ojo de la contemplación mediante el cual se accede a las realidades trascendentes.

Este enfoque surge de amplias investigaciones realizadas por el filósofo americano Ken Wilber, en las que, fundamentándose en diversos aportes de la psicología, la biología, la filosofía, la religión y la física emerge una forma integrada del conocimiento, así como una percepción coherente y sustentada del ser humano parte de lo que él llama Kosmos. La misma es abordada, no solo desde la perspectiva de la ciencia sino también desde la perspectiva de la religión místico-trascendental. En este sentido, Wilber (2008) plantea que "desde la «revolución cuántica» de hace cincuenta años, varios físicos han descubierto intrigantes paralelismos entre sus resultados y los de ciertas religiones místico-trascendentales", siendo cada vez más comunes 
y evidentes estas analogías; el autor menciona varios casos como por ejemplo el de Alan Watts quien para explicar el budismo y el taoísmo hizo uso de la física moderna y de la teoría de sistemas. (p.9)

Este enfoque epistémico provee las herramientas necesarias para abordar la realidad científica y tecnológica como proceso socio cultural de una manera eficaz y auténtica, respetando la importancia de cada persona, elemento y circunstancia que lo conforman; teniendo la posibilidad de descubrir la dinámica real del sujeto con el fenómeno, por ejemplo las TIC, en las diferentes etapas o roles que le toca asumir en el contexto investigado, pero además desde sus conocimientos, sus creencias, sus sueños y sus expectativas sin pasar por alto su entorno.

\section{LA IMAGINACIÓN, EL PRELUDIO DE LA VERDAD CIENTÍFICA}

Todos los avances que se han logrado a través de la historia en gran medida se deben a la imaginación del hombre, a esa posibilidad de poder manipular en su mente lo que sabe y lo que no, y armar nuevas conjeturas; nuevas hipótesis. Y el afán de que ese producto de la imaginación se convierta en realidad ha llevado a muchos a la consagración por los aportes que han dado a la humanidad. La imaginación científica viene a combinar, pues, los presupuestos teóricos y las vivencias cotidianas en el planteamiento de nuevas hipótesis, en el surgimiento de nuevas teorías, en el surgimiento de nuevas creaciones.

Ejemplos interesantes sobre imaginación científica son los diversos avances tecnológicos; siendo más precisos en este caso las TIC. Hace pocos años era ciencia ficción pensar que las personas se comunicasen a través de una pantalla. Algo que en los años 60 era solo imaginación, gracias a los avances científicos y tecnológicos, en la actualidad es parte de nuestra cotidianidad. En el diseño e implementación de este modo de comunicación se conjugaron aspectos empíricos y analíticos, los empíricos que vienen a ser el sonido y la electricidad, ambos medibles; por otra parte, los cálculos físicos y matemáticos necesarios para lograr la proyección de imagen y sonido a través de impulsos eléctricos conformarían el componente analítico.

Por otra parte, el campo de las ciencias sociales no escapa de la dinámica de la imaginación científica; las situaciones que han surgido de la dinámica social derivada del uso de las TIC inevitablemente han generado confrontaciones teóricas, ya que han conllevado al surgimiento de nuevas concepciones como es el caso de la Sociedad Red planteada por Castells (1999). Siendo ésta solo una en la gama de conjeturas que surgen del análisis de situaciones reales y posibles escenarios entramados en la imaginación de los investigadores sociales, las cuales ayudan a la comprensión de muchos fenómenos que han ido surgiendo con el uso de las TIC en los diversos campos de la vida cotidiana.

\section{REFLEXIONES FINALES}

El desarrollo de la ciencia, así como el de la tecnología ha estado influenciado por intereses diversos. El patrocinio o respaldo de proyectos científicos y tecnológicos muchas veces está condicionado al beneficio que connota el desarrollo de los mismo en campos como el político, económico o militar. Así pues, la verdad interesa por una necesidad de poder; de ahí la importancia de los criterios de validez, por los que la ciencia ha ido evolucionando y con los que, a través de sus métodos se ha logrado ampliar el abanico de posibilidades en las cuales cimentar la verdad desde diferentes visiones y con diferentes finalidades.

La verdad es un sinfín de destellos que se han producido paulatinamente a lo largo de la historia. La verdad, al igual que las TIC, cambia de acuerdo a los intereses, a la perspectiva, al enfoque que se quiera dar. La intermitencia de la verdad es lo que la hace apasionante y hace necesarios los criterios de validez científica, con los que nos permitimos consolidar, ese destello -esa verdad- que a uno a varios interesa resaltar.

\section{REFERENCIAS}

Adell, J. (1997). Tendencias en Educación en la Sociedad de la Información. Revista Electrónica de Tecnología Educativa. [Revista en línea], 7. Recuperado de: http://www.uib.es/depart/gte/revelec7.html

Bergua, J. (1970). Crítica de la Razón Pura, Kant. (Traducción, noticia preliminar y notas de Juan Bergua). España: Ediciones lbéricas. 
Díaz, L. (2011). Visión investigativa en ciencias de la salud (Énfasis en paradigmas emergentes). Valencia. Venezuela: Trotsky Vargas - Luis García.

Dunayevskaya, R. (2004). Filosofía y Revolución: De Hegel a Sartre y de Marx a Mao. México: Siglo XXI Editores.

Fernández, S. (1997). Habermas y la Teoría Crítica de la Sociedad. Legado y diferencias en teoría de la comunicación. Cinta de Moebio: Revista de epistemología de Ciencias Sociales. Recuperado de: http://www2.facso.uchile.cl/publicaciones/moebio/01/ frprin03.htm

Hurtado, I. y Toro, J. (2005). Paradigmas y métodos de investigación en tiempos de cambio. 5ta. Ed. Valencia, Venezuela: Episteme Consultores Asociados, C.A.

Mardones, (1991). Filosofía de las Ciencias humanas y sociales: Materiales para una fundamentación científica. España: Anthropos

Moreno, M. (2003). Filosofía. Volumen IV: Historia de la filosofía moderna y contemporánea. España: Editorial Mad, S.L.

Moya, E. (1998). La disputa del positivismo en la filosofía contemporánea. España: Servicio de publicaciones Universidad de Murcia.

Muñoz, B. (2000). Theodor W. Adorno: Teoría crítica y cultura de masas. Caracas: Fundamentos

Pérez, J (2006). Retórica, argumentación y elección de teorías en T. S. Kuhn. Caracas: Equinoccio: Universidad Simón Bolívar.

Prieto, M. (Dir.) (2002). Nietzche, Friedrich: El crepúsculo de los Ídolos. Buenos Aires: Edaf.

Selsam, H. (1962). ¿Qué es la filosofía? 3ra. Ed. México: Grijalbo, S.A.

Serrano, J. (2007). Filosofía actual en perspectiva latinoamericana. Bogotá: San Pablo.

Ulin, R. (1990). Antropología y teoría social. México: Siglo XXI Editores, S.A.

Velilla. M. (Comp.). (2002). Manual de iniciación pedagógica al pensamiento complejo. Colombia: Instituto colombiano de fomento para la educación superior/UNESCO

Wilber, K. (2006). Los tres ojos del conocimiento: la búsqueda de un nuevo paradigma. 5ta ed. Barcelona-España: Editorial Kairós, S.A.

Wilber, K.(Ed.) (2008). El paradigma holográfico: una exploración en las fronteras de la ciencia. 7ma ed. Barcelona-España: Editorial Kairós, S.A.

Fecha de recepción: 27/10/2019

Fecha de aceptación: 28/11/2019 